Published in final edited form as:

Sleep Med Clin. 2018 March ; 13(1): 13-19. doi:10.1016/j.jsmc.2017.09.002.

\title{
Insomnia in the Older Adult
}

Glenna Brewster, PhD, CRNP ${ }^{1,2}$, Barbara Riegel, PhD, RN ${ }^{1}$, and Philip R Gehrman, PhD, CBSM $^{2,3}$

${ }^{1}$ School of Nursing, Perelman School of Medicine of the University of Pennsylvania, Philadelphia, PA, USA

${ }^{2}$ Center for Sleep and Circadian Neurobiology, Perelman School of Medicine of the University of Pennsylvania, Philadelphia, PA, USA

${ }^{3}$ Department of Psychiatry, Perelman School of Medicine of the University of Pennsylvania, Philadelphia, PA, USA

\section{Keywords}

Sleep onset latency; sleep efficiency; benzodiazepines; sleep diary; pharmacotherapy; cognitive behavioral therapy for insomnia (CBTi); wake after sleep onset

\section{Introduction}

\section{Prevalence and diagnosis of insomnia}

Sleep changes with aging. Specifically, babies sleep between 10 to 14 hours per day while the recommended sleep duration for older adults is between $7-8$ hours daily. ${ }^{1}$ Many older adults experience dissatisfaction with the quantity and quality of sleep even with adequate opportunity to sleep; when this is accompanied by daytime impairment over a period of time, they may meet criteria for insomnia disorder (Table 1). Compared to younger adults, the prevalence of insomnia is higher in middle and older adults. ${ }^{2,3}$ and increases with age. Up to $50 \%$ of older adults report insomnia symptoms; however, this does not mean that insomnia is a normal part of aging. ${ }^{4}$

Sleep onset or initial insomnia is manifested by difficulty falling asleep that occurs at the start of the sleep period. ${ }^{5-7}$ Sleep maintenance or middle insomnia involves multiple and prolonged awakenings during the night. ${ }^{5-7}$ Late insomnia or early-morning awakenings is waking up early on mornings and being unable to return to sleep. ${ }^{5-7}$ Older adults tend to

CORRESPONDING AUTHOR. Dr. Glenna Brewster, 3624 Market Street, Suite 205, Philadelphia PA 19104,

glennasb@ nursing.upenn.edu.

3624 Market Street, Suite 205, Philadelphia PA 19104 glennasb@ nursing.upenn.edu

Room 418 Curie Blvd., 335 Fagin Hall, Philadelphia, Pennsylvania 19104, Tel: (215) 898-9927, briegel@ nursing.upenn.edu

3535 Market Street, Suite 670, Philadelphia, PA 19104. Tel \#: (215) 746-3578, gehrman@ exchange.upenn.edu

Publisher's Disclaimer: This is a PDF file of an unedited manuscript that has been accepted for publication. As a service to our customers we are providing this early version of the manuscript. The manuscript will undergo copyediting, typesetting, and review of the resulting proof before it is published in its final citable form. Please note that during the production process errors may be discovered which could affect the content, and all legal disclaimers that apply to the journal pertain.

DISCLOSURE STATEMENT

The authors have no financial or commercial disclosures. 
have more challenges with sleep maintenance compared to younger adults, ${ }^{3,4,8}$ which results in reductions in total sleep time and sleep efficiency. ${ }^{8}$ Insomnia can also be situational, persistent, or recurrent. ${ }^{5}$ Situational insomnia is usually acute insomnia which lasts a few days or weeks and is associated with changes in the sleep schedule or the sleep environment. ${ }^{5,8}$ Life events such as retirement, hospitalizations, and new onset illnesses can precipitate situational insomnia. Usually when the event that triggers the insomnia is resolved, so too does the insomnia. If the insomnia doesn't resolve, it evolves into chronic insomnia. ${ }^{5}$

Recurrent insomnia is episodic and often returns with the occurrence of stressful life events. ${ }^{5}$

\section{Risk factors of Insomnia}

Multiple factors increase the risk for older adults developing insomnia. They include environmental, behavioral, medical, and social factors ${ }^{8}$ (Table 2). For example, older adults may change their usual bed and wake time after they retire. Also, older adults also tend to have more comorbid disorders and are using multiple medications which further increases their risk for sleep disturbances. ${ }^{2,3}$

\section{Evaluation of Insomnia}

Insomnia is diagnosed through a detailed clinical history taken from the patient and their bed partner. ${ }^{9}$ In order to diagnose the specific type of insomnia, it is important for clinicians to ask about the history of insomnia, insomnia symptoms, sleep-wake routines and patterns, other sleep-related symptoms, daytime functioning and consequences, and previous treatments. ${ }^{9,10}$ Older adults should also be asked about whether they snore or have leg discomfort. ${ }^{11}$ Clinicians need to also identify whether there are comorbid medical, substance, and/or psychiatric conditions impacting sleep ${ }^{10}$ using screening tests such as the Patient Health Questionnaire- $9,{ }^{12}$ the Geriatric Depression Scale, ${ }^{13}$ and the General Anxiety Disorder Questionnaire. ${ }^{14}$ It is also important to assess daytime activities, as older adults may be less active during the day and consequently spend more time napping or dozing

Subjective measures that can be used to evaluate sleep include sleep questionnaires, like the Pittsburgh Sleep Quality Index (PSQI) and the Insomnia Severity Index (ISI), and sleep diaries. The PSQI is a 19 item instrument that assesses sleep quality and disturbances over a 1-month interval. ${ }^{15}$ Scores can range between $0-21$ with a score of 5 or more suggesting poor sleep quality. ${ }^{15}$ The ISI is a 7-item questionnaire that assesses the nighttime symptoms and impact of insomnia over the previous month. ${ }^{16}$ Scores range from 0 to 28 , with values $>14$ suggestive of moderate to severe insomnia. ${ }^{16}$

Sleep diaries allow for the prospective tracking of an individual's sleep/wake patterns. They capture information like bedtime, time to fall asleep, number and duration of nightly awakenings, wake-up time, out-of-bed time, and times and duration of daytime naps or dozing. ${ }^{9,17}$ Sleep diaries may also include questions about sleep quality, and types and amounts of medications, caffeine, and alcohol consumed. ${ }^{9}$ Sleep diaries completed for approximately two weeks allows for the recognition of sleep patterns and variability. ${ }^{9}$ Older patients sometime shave difficulities completing sleep diaries due ot visual impairments, and large print is sometimes used to make it easier for older patients to complete sleep diaries. 
Objective assessments of sleep such as actigraphy and polysomnography are not necessary for routine diagnosis and assessment of insomnia, but they may be helpful to rule out other comorbid sleep disorders such as sleep-disordered breathing or circadian rhythm sleep wake disorders. ${ }^{18-20}$

\section{Treatments for Insomnia}

The goal of insomnia treatment is the improvement of sleep quality and/or quantity and reduction in insomnia-related daytime impairments. ${ }^{9}$ The patient should be involved in the development of the treatment plan and decisions about which treatment goals to pursue since buy-in from the patient is critical for success. ${ }^{21}$ The choice of treatment depends on the severity and duration of the insomnia symptoms, coexisting disorders, willingness of the patient to engage in behavioral therapies, and vulnerability of the patient to the adverse effects of medications. ${ }^{9}$ It is important to emphasize to patients that it is normal to have occasional nights of poor sleep during and after the completion of treatment so that they will have realistic expectations about treatment and cope better during treatment. ${ }^{21}$

Sleep diaries and questionnaires can be used to evaluate how the treatment is progressing and to determine when there has been sufficient improvement to warrant discontinuation of treatment. ${ }^{9}$ After treatment is discontinued, it is important to conduct periodic follow-ups to identify potential recurrence and precipitating events due to changes in health or lifestyle.

\section{Non-Pharmacologic Treatment Options}

Cognitive Behavioral Therapy for Insomnia (CBTi) is a multicomponent intervention involving cognitive and behavioral techniques like stimulus control therapy, sleep restriction therapy, relaxation training, cognitive restructuring, and sleep hygiene education. ${ }^{22}$ The goal of CBTi is to replace maladaptive thoughts and sleep habits and to reduce arousal associated with sleep. ${ }^{22}$ CBTi has produced both short-term and long-term improvement in sleep. ${ }^{23}$ Although CBTi is effective for insomnia, many healthcare providers are neither aware of the existence of CBTi nor know how to refer patients for treatment. ${ }^{24}$

- Stimulus control aims to strengthen the association between the bed/bedroom and sleep and to produce a consistent sleep-wake schedule. Instructions for stimulus control include: minimize napping; go to bed only when sleepy; get out of bed if unable to sleep; use the bed/bedroom only for sleep and sex; and wake up at the same time each day. ${ }^{22}$

- $\quad$ Sleep restriction is used to increase homeostatic drive for sleep in order to improve sleep quality. The time spent in bed is reduced to the actual sleep duration based on sleep diaries. This creates some mild sleep deprivation. The time in bed is then gradually increased until the individual achieves an optimal sleep duration. ${ }^{22}$ There is strong support for sleep restriction in older adults with insomnia. ${ }^{25}$ Sleep compression is an alternative technique to sleep restriction where time in bed is gradually reduced until the older adult achieves an optimal sleep duration. ${ }^{26}$ 
- Relaxation training is done to reduce tension and intrusive thoughts which interfere with the ability to sleep. Relaxation techniques used include deep breathing exercises, progressive muscle relaxation, biofeedback, and guided imagery. ${ }^{22}$

- $\quad$ Cognitive restructuring aims to reduce worry and change misconceptions associated with sleep and insomnia using Socratic questioning. ${ }^{22}$ Challenging inaccurate patterns of thinking can change in how older adults perceive the effect of sleep on their lives.

- $\quad$ Sleep hygiene education provides some guidelines about factors that may help or interfere with sleep. They include: not eating a heavy meal or drinking alcohol within two hours of bedtime; limiting caffeine intake after lunchtime; exercising regularly but not within 2 hours of bedtime; and keeping the bedroom quiet, dark and at a cool, comfortable temperature. ${ }^{22}$

Mindfulness-based stress reduction techniques aim to change reactions to stress by teaching purposeful awareness and acceptance of the present state. ${ }^{27}$ and includes techniques such as breathing, body scan, and walking meditations, and Hatha Yoga. ${ }^{28}$ These techniques have been effective in reducing insomnia in older adults. ${ }^{28}$

Bright light therapy helps to strengthen circadian rhythms and establish a healthy sleep-wake cycle. ${ }^{29,30}$ Results have been mixed on its efficacy for insomnia treatment of insomnia in older adults ${ }^{29,30}$ but in general, may have a favorable effect when used with older adults. ${ }^{29}$ Healthcare practitioners provide white light sources with a bluish tint that provide at least 1,000 lux at the eye during daytime hours; at a time that is most convenient for the patient; on mornings after awakening if the circadian timing of the patient is unknown; or during the time interval the patient tends to be more tired. ${ }^{29}$

Acupuncture is a traditional Chinese technique in which specific points on the body are stimulated usually by inserting thin needles through the skin. ${ }^{31}$ It has been shown to be effective in improving insomnia symptoms in older adults ${ }^{31}$

\section{Pharmacologic Treatment Options}

Benzodiazepines, such as Lorazepam, Temazepam, and Clonazepam, decrease sleep latency and decrease nocturnal awakenings, but they also reduce rapid eye movement sleep. ${ }^{32}$ in older adults, they increase the risk for memory impairment, falls, fractures and motor vehicle accidents and avoidable emergency department visits and hospital admissions; therefore their use should be avoided in older adults. ${ }^{33,34}$ Between $5.3 \%$ to $10.8 \%$ of adults 50 years and older use benzodiazepines. ${ }^{35}$ Long-term use of benzodiazepines can promote psychological dependence and there is an increased risk of addiction and abuse. ${ }^{36}$ Tolerance can also develop over time thus requiring larger doses to sustain efficacy. ${ }^{36}$ It is necessary to educate older adults about the effects of benzodiazepine use and encourage discontinuation through tapering. ${ }^{33,37}$ While there is no current standard for tapering benzodiazepines, some providers recommend establishing a longer tapering schedule over 4-5 months for older patients and tapering the dose by $25 \%$ every 2 weeks. ${ }^{33,37,38}$ While tapering healthcare providers could provide cognitive behavioral therapy for insomnia. ${ }^{37,38}$ 
Non-benzodiazepine hypnotics reduce sleep latency. ${ }^{32}$ These medications which include Eszopiclone, Zolpidem and Zaleplon should be avoided in older adults without consideration of the duration of use (no more than 90 days) because they can cause confusion and they increase the risk of falls and fractures. This should be avoided in older adults with dementia and cognitive impairment. ${ }^{34}$

Melatonin receptor agonists reduce sleep latency and increased sleep duration. ${ }^{391} \mathrm{An}$ example is Ramelteon. Potential adverse effects include mild gastrointestinal disturbances and nervous system effects such as dizziness, headache, somnolence, and fatigue with no evidence of significant rebound insomnia or withdrawal effects ${ }^{39}$ Ramelteon has been shown to decrease sleep latency in older adults. ${ }^{40}$

Many antidepressants have sedating effects and are sometimes used to treat insomnia, often at lower doses than used for depression. Antidepressants often have overall REMsuppressing effects, can decrease slow-wave sleep latency, and duration of slow wave sleep. ${ }^{32}$ In the absence of an underlying depressive disorder, antidepressants should also be avoided in older adults because they are highly anticholinergic, sedating, increase the risk of falls, and cause orthostatic hypotension. ${ }^{34}$

Antihistamines decrease sleep latency; ${ }^{32}$ however, these over-the-counter sleep medications like diphenhydramine, produce rapid tolerance and are highly anticholinergic. ${ }^{34}$

Anticholinergic effects include blurred vision, dizziness, difficulty urinating, dry mouth and constipation. Anticholinergic medications can also increase the risk for cognitive impairment and decline; therefore drugs with a high anticholinergic profile such as antihistamines, antidepressants should be avoided in older adults. ${ }^{34}$

Melatonin and Valerian Root are classified as complementary and alternative medications. They are supplements and are not regulated by the Food and Drug Administration. The doses and the preparations available to consumers usually vary significantly. Melatonin is an endogenous hormone secreted by the pineal gland but is also used as an exogenous supplement. ${ }^{41,42}$ Melatonin decreases subjective sleep latency in some studies, although it may cause headaches and drowsiness. ${ }^{41,42}$ Valerian root has been shown to improve subjective sleep parameters but the research is less consistent with objective sleep parameters. Rare side effects reported for valerian root include gastrointestinal upset, contact allergies, headache, and restless sleep. ${ }^{41}$ Individuals using complementary and alternative medications should always inform their healthcare provider and consider the interactions between herbal remedies and prescription medications. ${ }^{41}$

Pharmacologic treatments should only be used for short-term management of insomnia. When pharmacotherapy is used, healthcare providers should consider the insomnia symptoms, whether other treatments are available, how the patient responded to previous treatments, the side effect profile of the medication, and medication interactions. ${ }^{36}$ Older adults have better response rates with lower dosages because decreased lean body mass, increased body fat, and reduction in plasma proteins may increase blood concentration of unbound drugs and drug halflife. ${ }^{36}$ Therefore, when prescribing medications for insomnia for older adults, start with the lowest dose and titrate upwards. ${ }^{36}$ Given the side effects and 
concerns about long-term safety, it is recommended that pharmacotherapy for insomnia be avoided or be used for only for short periods of time. ${ }^{34,43}$

\section{Comparative effectiveness of CBT-I and Medications}

A comparative effectiveness study of CBTi compared to sleep medications (zopiclone, zolpidem, temazepam, or triazolam) found that CBTi is as effective for short-term treatment of insomnia as medications. ${ }^{24,44}$ The effects of CBTi may also be more long-lasting than medications. ${ }^{22,24}$ While medications produce more rapid improvements compared to $\mathrm{CBTi}$, over the long-term, CBTi has more durable and sustained effects on sleep quality and outcomes. The therapeutic effects of medications are usually not maintained after the medication is discontinued. ${ }^{24}$

\section{Summary}

Aging is associated with a number of changes in sleep continuity and architecture parameters. Many older adults experience difficulties with falling asleep, staying asleep, or waking up too early which leads to daytime impairment and warrant a diagnosis of insomnia. Factors such as the sleep environment, medications, and medical and psychiatric disorders can increase the risk for insomnia. Therefore, healthcare providers should obtain a comprehensive health and sleep history from older adults in order to correctly diagnose insomnia and identify the potential correlates of the disorder. After diagnosing insomnia, first line treatment is CBTi. Medications such as benzodiazepines and non-benzodiazepines should be avoided in older adults given their potential for significant adverse consequences and clinical guidelines recommending against their use.

\section{Acknowledgments}

Glenna Brewster is a Ruth L. Kirschstein NRSA Postdoctoral Research Fellow (T32HL07713; PI: Pack, A.)

\section{References}

1. Hirshkowitz M, Whiton K, Albert SM, et al. National Sleep Foundation's sleep time duration recommendations: methodology and results summary. Sleep Health. 2015; 1(1):40-43. [PubMed: 29073412]

2. Blay SL, Andreoli SB, Gastal FL. Prevalence of self-reported sleep disturbance among older adults and the association of disturbed sleep with service demand and medical conditions. International psychogeriatrics. Jun; 2008 20(3):582-595. [PubMed: 18053289]

3. Leblanc M-F, Desjardins S, Desgagné A. Sleep problems in anxious and depressive older adults. Psychology Research and Behavior Management. Jun 11.2015 8:161-169. [PubMed: 26089709]

4. Ohayon MM. Epidemiology of insomnia: what we know and what we still need to learn. Sleep medicine reviews. Apr; 2002 6(2):97-111. [PubMed: 12531146]

5. Sleep-Wake Disorders. Diagnostic and Statistical Manual of Mental Disorders.

6. Lichstein KL, Durrence HH, Taylor DJ, Bush AJ, Riedel BW. Quantitative criteria for insomnia. Behav Res Ther. Apr; 2003 41(4):427-445. [PubMed: 12643966]

7. Lineberger MD, Carney CE, Edinger JD, Means MK. Defining insomnia: quantitative criteria for insomnia severity and frequency. Sleep. Apr; 2006 29(4):479-485. [PubMed: 16676781]

8. Vitiello MV. Sleep in Normal Aging. Sleep Medicine Clinics. 7(3):539-544. 
9. Schutte-Rodin S, Broch L, Buysse D, Dorsey C, Sateia M. Clinical Guideline for the Evaluation and Management of Chronic Insomnia in Adults. Journal of Clinical Sleep Medicine. 2008; 4(5):487504. [PubMed: 18853708]

10. Mai E, Buysse DJ. Insomnia: Prevalence, Impact, Pathogenesis, Differential Diagnosis, and Evaluation. Sleep medicine clinics. 2008; 3(2):167-174. [PubMed: 19122760]

11. McCall WV. Sleep in the Elderly: Burden, Diagnosis, and Treatment. Primary Care Companion to The Journal of Clinical Psychiatry. 2004; 6(1):9-20.

12. Kroenke K, Spitzer RL, Williams JBW. The PHQ-9: Validity of a Brief Depression Severity Measure. Journal of General Internal Medicine. 2001; 16(9):606-613. [PubMed: 11556941]

13. Yesavage JA, Brink TL, Rose TL, et al. Development and validation of a geriatric depression screening scale: a preliminary report. Journal of psychiatric research. 1982; 17(1):37-49. [PubMed: 7183759]

14. Wild B, Eckl A, Herzog W, et al. Assessing generalized anxiety disorder in elderly people using the GAD-7 and GAD-2 scales: results of a validation study. The American journal of geriatric psychiatry. Oct; 2014 22(10):1029-1038. [PubMed: 23768681]

15. Buysse DJ, Reynolds CF 3rd, Monk TH, Berman SR, Kupfer DJ. The Pittsburgh Sleep Quality Index: a new instrument for psychiatric practice and research. Psychiatry research. May; 1989 28(2):193-213. [PubMed: 2748771]

16. Bastien $\mathrm{CH}$, Vallieres A, Morin CM. Validation of the Insomnia Severity Index as an outcome measure for insomnia research. Sleep medicine. 2001; 2(4):297-307. [PubMed: 11438246]

17. Carney CE, Buysse DJ, Ancoli-Israel S, et al. The Consensus Sleep Diary: Standardizing Prospective Sleep Self-Monitoring. Sleep. 2012; 35(2):287-302. [PubMed: 22294820]

18. Littner M, Hirshkowitz M, Kramer M, et al. Practice parameters for using polysomnography to evaluate insomnia: an update. Sleep. Sep; 2003 26(6):754-760. [PubMed: 14572131]

19. Ancoli-Israel S, Cole R, Alessi C, Chambers M, Moorcroft W, Pollak CP. The role of actigraphy in the study of sleep and circadian rhythms. Sleep. May 1; 2003 26(3):342-392. [PubMed: 12749557]

20. Roehrs T. Sleep physiology and pathophysiology. Clinical cornerstone. 2000; 2(5):1-15.

21. Gooneratne NS, Vitiello MV. Sleep In Older Adults: Normative Changes, Sleep Disorders, and Treatment Options. Clinics in geriatric medicine. Jun 12; 2014 30(3):591-627. [PubMed: 25037297]

22. Morin CM, Bootzin RR, Buysse DJ, Edinger JD, Espie CA, Lichstein KL. Psychological and behavioral treatment of insomnia:update of the recent evidence (1998-2004). Sleep. Nov; 2006 29(11):1398-1414. [PubMed: 17162986]

23. Alessi C, Martin JL, Fiorentino L, et al. Cognitive Behavioral Therapy for Insomnia in Older Veterans Using Nonclinician Sleep Coaches: Randomized Controlled Trial. Journal of the American Geriatrics Society. 2016; 64(9):1830-1838. [PubMed: 27550552]

24. Mitchell MD, Gehrman P, Perlis M, Umscheid CA. Comparative effectiveness of cognitive behavioral therapy for insomnia: a systematic review. BMC Family Practice. 2012; 13:40-40. [PubMed: 22631616]

25. McCurry SM, Logsdon RG, Teri L, Vitiello MV. Evidence-based psychological treatments for insomnia in older adults. Psychology and aging. Mar; 2007 22(1):18-27. [PubMed: 17385979]

26. Lichstein KL, Riedel BW, Wilson NM, Lester KW, Aguillard RN. Relaxation and sleep compression for late-life insomnia: a placebo-controlled trial. Journal of consulting and clinical psychology. Apr; 2001 69(2):227-239. [PubMed: 11393600]

27. Ong JC, Manber R, Segal Z, Xia Y, Shapiro S, Wyatt JK. A Randomized Controlled Trial of Mindfulness Meditation for Chronic Insomnia. Sleep. 2014; 37(9):1553-1563. [PubMed: 25142566]

28. Zhang JX, Liu XH, Xie XH, et al. Mindfulness-based stress reduction for chronic insomnia in adults older than 75 years: a randomized, controlled, single-blind clinical trial. Explore (New York, N.Y.). May-Jun;2015 11(3):180-185.

29. Sloane PD, Figueiro M, Cohen L. Light as Therapy for Sleep Disorders and Depression in Older Adults. Clinical geriatrics. 2008; 16(3):25-31. [PubMed: 24285919] 
30. Gammack JK. Light therapy for insomnia in older adults. Clin Geriatr Med. Feb; 2008 24(1):139149. viii. [PubMed: 18035237]

31. Kwok T, Leung PC, Wing YK, et al. The effectiveness of acupuncture on the sleep quality of elderly with dementia: a within-subjects trial. Clinical Interventions in Aging. Jul 31.2013 8:923929. [PubMed: 23940415]

32. Pagel JF, Parnes BL. Medications for the Treatment of Sleep Disorders: An Overview. Primary Care Companion to The Journal of Clinical Psychiatry. 2001; 3(3):118-125.

33. Tannenbaum C. Inappropriate benzodiazepine use in elderly patients and its reduction. Journal of Psychiatry \& Neuroscience. 2015; 40(3):E27-E28. [PubMed: 25903036]

34. By the American Geriatrics Society Beers Criteria Update Expert P. American Geriatrics Society 2015 Updated Beers Criteria for Potentially Inappropriate Medication Use in Older Adults. Journal of the American Geriatrics Society. 2015; 63(11):2227-2246. [PubMed: 26446832]

35. Olfson M, King M, Schoenbaum M. Benzodiazepine use in the united states. JAMA Psychiatry. 2015; 72(2):136-142. [PubMed: 25517224]

36. Kamel NS, Gammack JK. Insomnia in the elderly: cause, approach, and treatment. Am J Med. Jun; 2006 119(6):463-469. [PubMed: 16750956]

37. Bélanger L, Belleville G, Morin C. Management of Hypnotic Discontinuation in Chronic Insomnia. Sleep medicine clinics. 2009; 4(4):583-592. [PubMed: 20607118]

38. Paquin AM, Zimmerman K, Rudolph JL. Risk versus risk: a review of benzodiazepine reduction in older adults. Expert opinion on drug safety. Jul; 2014 13(7):919-934. [PubMed: 24905348]

39. Roth T, Seiden D, Sainati S, Wang-Weigand S, Zhang J, Zee P. Effects of ramelteon on patientreported sleep latency in older adults with chronic insomnia. Sleep medicine. 7(4):312-318.

40. Roth T, Seiden D, Sainati S, Wang-Weigand S, Zhang J, Zee P. Effects of ramelteon on patientreported sleep latency in older adults with chronic insomnia. Sleep medicine. Jun; 2006 7(4):312318. [PubMed: 16709464]

41. Gooneratne NS. Complimentary and Alternative Medicine for Sleep Disturbances in Older Adults. Clinics in geriatric medicine. 2008; 24(1):121-viii. [PubMed: 18035236]

42. Buscemi N, Vandermeer B, Hooton N, et al. The Efficacy and Safety of Exogenous Melatonin for Primary Sleep Disorders: A Meta-Analysis. Journal of General Internal Medicine. 2005; 20(12): 1151-1158. [PubMed: 16423108]

43. Perlis M, Gehrman P, Riemann D. Intermittent and long-term use of sedative hypnotics. Current pharmaceutical design. 2008; 14(32):3456-3465. [PubMed: 19075721]

44. Sivertsen B, Omvik S, Pallesen S, et al. Cognitive behavioral therapy vs zopiclone for treatment of chronic primary insomnia in older adults: A randomized controlled trial. JAMA. 2006; 295(24): 2851-2858. [PubMed: 16804151]

45. American Academy of Sleep Medicine. International classification of Sleep Disorders. 3. Darien, IL: American Academy of Sleep Medicine; 2014. 


\section{KEY POINTS}

- The incidence of insomnia increases with aging. Insomnia can include difficulty falling asleep at the start of the sleep period, waking up during the night and having difficulty falling back asleep, and waking up early and being unable to get back to sleep. Difficulty staying asleep and early morning insomnia are common in older adults with insomnia disorder.

- When diagnosing insomnia, healthcare providers need to collect a thorough health history and include questions about the older adult's sleep, medical, and psychiatric history.

- $\quad$ Cognitive Behavioral Therapy for Insomnia, which consists of stimulus control, sleep restriction, sleep hygiene and cognitive therapy, is the recommended first-line therapy for treatment of insomnia in older adults.

- $\quad$ Because of higher risk for adverse effects in older patients, medications should be used sparingly, and when possible, be discontinued.

- Cognitive behavioral therapy for insomnia has been shown to be more efficacious than medications for the long-term management of insomnia in older adults. 


\section{SYNOPSIS}

While insomnia is not a normal part of the aging process, its prevalence increases with age. Factors such as medications and medical and psychiatric disorders can increase the risk for insomnia. In order to diagnose insomnia, it is important for older adults to complete comprehensive sleep and health histories. Cognitive Behavioral Therapy for Insomnia, which includes stimulus control, sleep restriction, sleep hygiene, and cognitive therapy, is the recommended first-line treatment for insomnia and is more effective that medications for the long-term management of insomnia. Medications such as benzodiazepines and antidepressants should be avoided for the treatment of insomnia in older adults. 
Table 1

\section{Diagnostic Criteria for Insomnia}

\begin{tabular}{|c|c|c|}
\hline \multicolumn{3}{|c|}{ Diagnostic criteria for chronic insomnia (ICSD-3) ${ }^{45}$} \\
\hline \multicolumn{3}{|c|}{ Criteria A-F must be met } \\
\hline \multirow[t]{6}{*}{ A. } & $\begin{array}{l}\text { The } \\
\text { obse }\end{array}$ & $\begin{array}{l}t \text { reports, or the patient's parent or caregiver } \\
\text { one or more of the following: }\end{array}$ \\
\hline & 1. & Difficulty initiating sleep \\
\hline & 2. & Difficulty maintaining sleep \\
\hline & 3. & Waking up earlier than desired \\
\hline & 4. & Resistance to going to bed on appropriate schedule \\
\hline & 5. & $\begin{array}{l}\text { Difficulty sleeping without parent or caregiver } \\
\text { intervention }\end{array}$ \\
\hline
\end{tabular}

B. The patient reports, or the patient's parent or caregiver observes, one or more of the following related to the nighttime sleep difficulty:

1. Fatigue/malaise

2. Attention, concentration or memory impairment

3. Impaired social, family, occupational or academic performance

4. Mood disturbance/irritability

5. Daytime sleepiness

6. Behavioral problems (e.g. hyperactivity, impulsivity, aggression)

7. Reduced motivation/energy/initiative

8. Proneness for errors/accidents

9. Concerns about or dissatisfaction with sleep

C. The reported sleep/wake complaints cannot be explained purely by inadequate opportunity (i.e. enough time is allotted for sleep) or inadequate circumstances (i.e. the environment is safe, dark, quiet and comfortable) for sleep.

D. The sleep disturbance and associated daytime symptoms occur at least three times per week

E. The sleep disturbance and associated daytime symptoms have been present for at least 3 months

F. The sleep/wake difficulty is not explained more clearly by another sleep disorder
Diagnostic criteria for chronic insomnia (DSM-5) ${ }^{5}$

$307.42(\mathrm{~F} 51.01)$

1 A predominant complaint of dissatisfaction with sleep quantity or quality, associated with one (or more) of the following symptoms:

1. Difficulty initiating sleep. (In children, this may manifest as difficulty initiating sleep without caregiver intervention.)

2. Difficulty maintaining sleep, characterized by frequent awakenings or problems returning to sleep after awakenings. (In children, this may manifest as difficulty returning to sleep without caregiver intervention.)

3. Early-morning awakening with inability to return to sleep.

2 The sleep disturbance causes clinically significant distress or impairment in social, occupational, educational, academic, behavioral, or other important areas of functioning.

3 The sleep difficulty occurs at least 3 nights per week.

4 The sleep difficulty is present for at least 3 months.

5 The sleep difficulty occurs despite adequate opportunity for sleep.

6 The insomnia is not better explained by and does not occur exclusively during the course of another sleepwake disorder (e.g., narcolepsy, a breathing-related sleep disorder, a circadian rhythm sleep-wake disorder, a parasomnia).

7 The insomnia is not attributable to the physiological effects of a substance (e.g., a drug of abuse, a medication).

8 Coexisting mental disorders and medical conditions do not adequately explain the predominant complaint of insomnia.

Specify if:

- $\quad$ Episodic: Symptoms last at least 1 month but less than 3 months.

- Persistent: Symptoms last 3 months or longer.

- Recurrent: Two (or more) episodes within the space of 1 year. 


\section{Table 2}

Risk Factors for Insomnia in Older Adults $2,5,8,9,34$

\begin{tabular}{|c|c|}
\hline Environment & $\begin{array}{l}\text { Excessive noise, hot or cold temperatures, light during the sleep period, } \\
\text { Moving to a new home or downsizing to a smaller space or a retirement community or related facility } \\
\text { Institutionalization }\end{array}$ \\
\hline Behavioral/Social & $\begin{array}{l}\text { Irregular sleep schedules, caffeine use later in the day, alcohol close to bedtime. } \\
\text { Caregiving, hospitalizations, new medical problems } \\
\text { Retirement or lifestyle change } \\
\text { Death of a family member or friend } \\
\text { Inappropriate use of social drugs, e.g. alcohol (Note that alcohol is frequently used to self-medicate for sleep problems. It } \\
\text { helps with falling asleep; however, when the effect wears off, sleep becomes light and disrupted.) } \\
\text { Napping }\end{array}$ \\
\hline Demographics & Female gender \\
\hline Medical & $\begin{array}{l}\text { Medications: Theophylline, thyroid hormone, anti-cholinergics, stimulants, oral decongestants, antidepressants, } \\
\text { corticosteriods, antihypertensives, opioids, non-steroidal anti-inflammatory drugs } \\
\text { Sleep disorders: Sleep apnea, restless leg syndrome, periodic limb movement disorder, rapid eye movement disorder, Age- } \\
\text { related circadian rhythm change (phase advance) } \\
\text { Psychiatric and cognitive conditions Depression, anxiety, mania, panic attacks, schizophrenia, substance abuse, dementia } \\
\text { Other Medical conditions: diabetes, fibromyalgia, hypertension, cardiovascular disease, stroke, chronic pain }\end{array}$ \\
\hline
\end{tabular}

